\title{
Aging and gut brain axis
}

\begin{abstract}
The rapidly aging population in this century, with the accompanying age related disorders is a medical, psychosocial and financial burden on the society. This maturing of the population has been correlated with altered gut brain axis and chronic inflammatory state. The gut inflammatory reactions with release of pro-inflammatory metabolites are cause or/and consequence of age related disorders in elderly. Therefore, dietary microbiome therapies such as probiotic and prebiotics supplementation may induce beneficial changes in diversity and taxa of the gut microbiome resulting in healthy ageing. To summarize, research in this field is the need of the hour to understand senescence vis -a- vis gut brain axis.
\end{abstract}

Keywords: gut brain axis, aging, microbiome, probiotic, prebiotic, inflammatory state
Volume 6 Issue 2 - 2021

\section{Manjula Suri}

Head, Department of Physiology and Promotive Health, Institute of Home Economics, University of Delhi, India

Correspondence: Manjula Suri, Head, Department of Physiology and Promotive Health, Institute of Home Economics, University of Delhi, New Delhi, India, Email mnjlsuri@gmail.com

Received: February 18, 2021 | Published: April 21, 202 |

\section{Introduction}

Increased life expectancy in the 20th century is leading to a demographic shift resulting in population aging. It is a major medicosocial burden requiring the need for the diagnosis and the management of age related disorders. A large body of literature provide compelling evidence that a correlation exist between aging/ age-related chronic diseases with chronic inflammatory state..$^{1-3}$ Review of literature in the geriateric research shows two important hypotheses associated with age-related inflammation: inflammaging ${ }^{4,5}$ and molecular inflammation. ${ }^{6-10}$ Gut microbes could unlock the secret to healthy aging. Various researchers reported the bidirectional crosstalk between the brain and the gut with reciprocal influence on their functions and dysfunctions. Therefore, research is going on in the field of potential diagnostic and therapeutic role of the microbiota in improving the altered gut-brain axis in elderly patients and the use of specific stool bacteria as biomarkers for disease progression.

\section{Gut microbiota}

Gut microbiota are a community of commensals and pathogenic microorganisms present in our body ]. There exists a symbiotic relationship between the host organisms and the gut microbiota. Total microbial cells in the gut are actually more than the human cells in the body and weight of these gut microbes is approx.1-2 kg. ${ }^{11}$ The gut has about 100 trillions of microganisms - bacteria, yeasts, helminthes, viruses, and protozoa. The colonization of the gut microbiota commences at birth and the factors influencing microbiota composition include, mode of delivery, breastfeeding, infection, antibiotics, diet and stress. ${ }^{12}$

\section{Gut microbiota and brain crosstalk}

Communication between the different constituents of the microbiota-gut-brain axis has been well established by various clinical trials. Researchers observed that germ-free (GF) mice show an enhanced hypothalamus-pituitary axis (HPA) reaction to stress compared to a normal mouse. Nobuyuki Sudo et al. ${ }^{13}$ reported that commensal microbiota are an environmental causal factor that modulates the development of the HPA response to stress. These findings substantiate that the serial events in the gut following postnatal microbial colonization can have a enduring effect on the processing of sensory information regarding the endocrine stress axis by the nervous system. A crosstalk between gut and brain was observed to be primarily relying on at least two factors i.e. vagus nerve signaling and specific bacterial species. Research based on in vivo findings, provides evidence of a novel link between indigenous microorganisms and the nervous system and shows a new aspect of the brain-gut axis ${ }^{14}$ such as

i. Ability to synthesize / mimic molecules e.g. acetylcholine, GABA, serotonin etc.

ii. Alteration of composition of gut microbiota due to emotional stress.

iii. Gut microbiota has significant effect on the development of emotional response, stress response, modulation of pain perception and functioning of neuromodulators.

iv. Gut mirobiota compostion influences high cognitive processes.

v. Microbiota-GBA interactions comes from the association of dysbiosis with central nervous disorders (i.e. autism, anxietydepressive behaviors) and functional gastrointestinal disorders (Figure 1).

Effect of aging on microbiome-gut brain axis is as follows:

a) Modification in composition of normal microflora of GIT e.g. commensals such as bacteroides, bifidobacteria and lactobacilli are found to be attenuated while the levels of opportunists such as enterobacteria, $C$. perfringens and $C$. difficile are augmented in elderly.

b) Chronic low grade inflammation(LGI) in elderly which is influenced by gut microbiota and diet.

c) Modified intestinal metabolism and gut microbiota could be targeted to improve metabolic diseases like obesity.

d) Reduced availability of neuromodulators or their precursors \& short chain fatty acids

e) Immunnosenescence and high susceptibility to infections

f) Increased intestinal porosity via age related redesigning of proteins present in intestinal epithelial tight junction.

g) Increased colonic cytokine expression/ inflammaging ${ }^{15}$ e.g.increased levels of TNF, IL- 6 and IL- 8 in old-age may be associated with changes in gut microbiota. ${ }^{16,17}$ 
h) Mechanism of alteration: In elderly altered diet, gut physiology, gut microbiota and a weak immune system results in gut inflammation with release of pro-inflammatory metabolites. ${ }^{18-20}$
These inflammatory reactions are related to neuroinflammation, activation of neuroglial cells and cognitive impairment in elderly. ${ }^{21-22}$

\section{Ageing and Gut brain Axis}

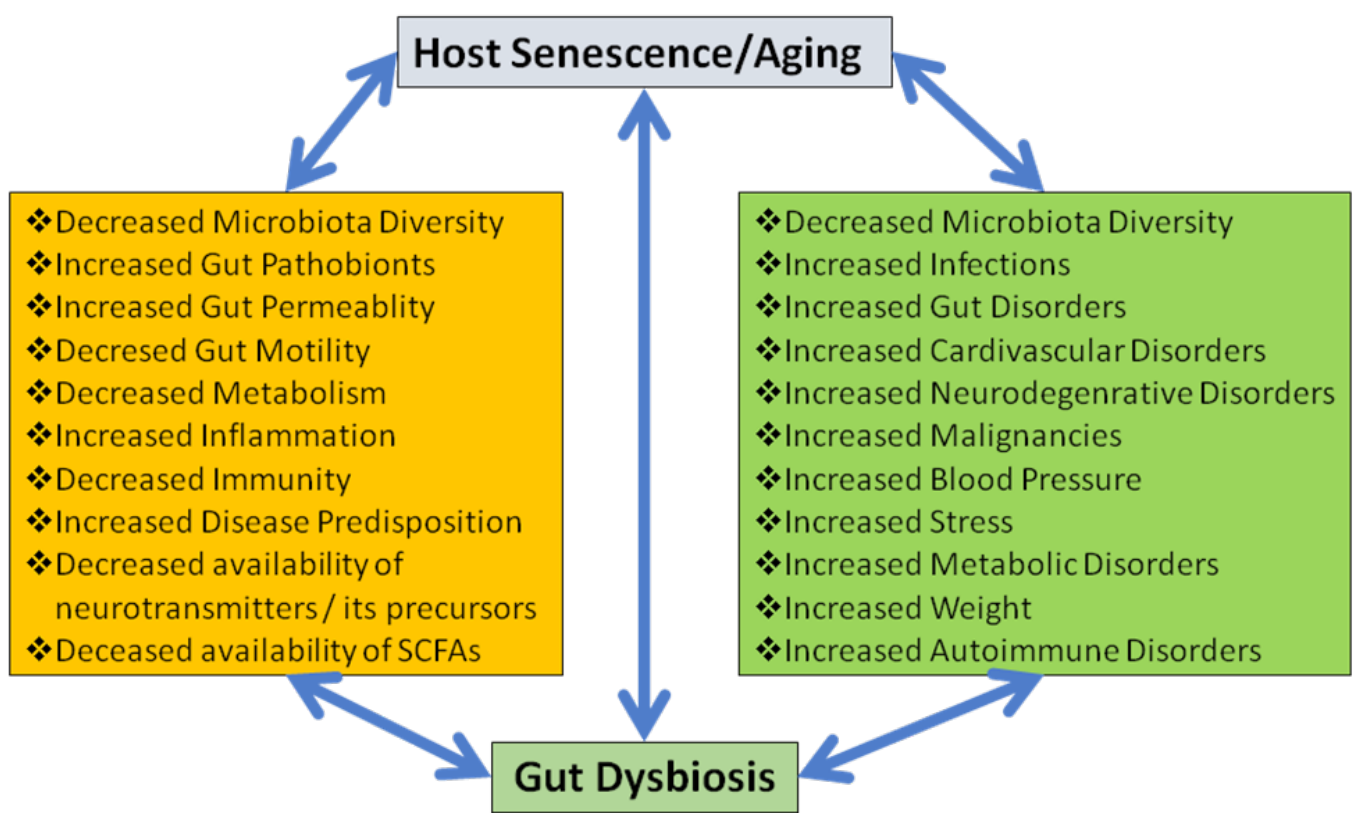

Figure I Altered gut brain axis and aging.

\section{Dietary control of gut brain axis in elderly}

Dietary interventions may have the inherent capacity to regulate age related symptoms associated with gut-brain axis dysfunction. Oxidative stress and inflammation act as the two primary causes of brain aging. Therefore use of antioxidants and anti-inflammatory molecules play a pivitol role in prevention of brain aging and brain age related diseases. ${ }^{23}$ Similarly prebiotics are characterized as nondigestible dietary ingredients that have beneficial effect on the host by selectively promote the growth and physiological functions of bacteria in colon. Fermentation of prebiotics generates short chain fatty acids (SCFAs) which exerts favorable impact on the integrity of epithelial barrier, but also on brain metabolism. SCFAs could ultimately be used as interventional substances to target microbiotagut-brain interactions in humans. ${ }^{24}$ Both prebiotics and probiotics may be helpful in malnutrition, constipation and immunity reduction in the elderly.

\section{Conclusion}

To conclude, a complete understanding of the mechanisms involved in the age-mediated changes in the gut brain axis, or whether these changes are the cause or/and consequence of senescence is not clearly understood. The aging process may indeed alter the gut microbiota and the brain. Recent studies are also exploring the effective ways to improve animal longevity through targeted microbiota alterations. ${ }^{25}$ But more research needs to be conducted to further elucidate the mechanisms of the gut-brain axis and aging.

\section{Acknowledgments}

None.

\section{Conflicts of interest}

The authors declare that there is no conflict of interest.

\section{References}

1. Chung HY, Cesari M, Anton S, et al. (2009). Molecular inflammation: underpinnings of aging and age-related diseases. Ageing Res Rev. 2009;8:18-30.

2. Maya EK, Ruslan. Inflammation and disease susceptibility. Cell. $2015 ; 160: 816-827$.

3. Franceschi C, Campisi J. Chronic inflammation (inflammaging) and its potential contribution to age-associated diseases. J Gerontol A Biol Sci Med Sci. 2014;69:S4-9.

4. Franceschi C, Bonafè M, Valensin S, et al. Inflamm-aging. An evolutionary perspective on immunosenescence. Ann N Y Acad Sci. 2000;908:244-254.

5. Franceschi C, Capri M, Monti D, et al. Inflammaging and antiinflammaging: a systemic perspective on aging and longevity emerged from studies in humans. Mech Ageing Dev. 2007;128:92-105.

6. Chung HY, Sung B, Jung KJ, et al. The molecular inflammatory process in aging. Antioxid Redox Signal. 2006;8:572-581.

7. Chung HY, Kim HJ, Jung KJ, et al. The inflammatory process in aging. Rev Clin Gerontol. 2000;10:207-222.

8. Chung HY, Kim HJ, Kim JW, et al.The inflammation hypothesis of aging: molecular modulation by calorie restriction. Ann N Y Acad Sci. 2001;928:327-335.

9. Chung HY, Kim HJ, Kim KW, et al. Molecular inflammation hypothesis of aging based on the anti-aging mechanism of calorie restriction. Microsc Res Tech. 2002;59:264-272. 
10. Chung HY, Lee EK, Choi YJ, et al. Molecular inflammation as an underlying mechanism of the aging process and age-related diseases. $J$ Dent Res. 2011;90:830-840.

11. Dinan TG, Cryan JF. Melancholic microbes: a link between gut microbiota and depression?. Neurogastroenterol Motil. 2013;25:713-9.

12. Barbara G, Stanghellini V, Brandi G, et al. Interactions between commensal bacteria and gut sensorimotor function in health and disease. Am J Gastroenterol. 2005;100:2560-2568.

13. Nobuyuki SUDO. Role of gut microbiota in brain function and stressrelated pathology. Biosci Microbiota Food Health. 2019;38(3): 75-80.

14. Nobuyuki Sudo, Yoichi Chida, Yuji Aiba, et al. Postnatal microbial colonization programs the hypothalamic-pituitary-adrenal system for stress response in mice. J Physiol. 2004;558(Pt 1):263-275.

15. Enciu A-M, Codrici E, Mihai S, et al. Role of Neutraceuticals in modulation of Gut Brain Axis in elderly person. Open access peer reviewed, edited book titled. Gerontology. 2018:10.5572.

16. Ouwehand AC, Bergsma N, Parhiala R, et al. Bifidobacterium microbiota and parameters of immune function in elderly subjects. FEMS Immunol Med Microbiol. 2008;53(1):18-25.

17. Biagi E, Nylund L, Candela M, et al. Through aging, and beyond: Gut microbiota and inflammatory status in seniors and centenarians. PloS One. 2010;5(5):e10667.
18. Maffei VJ, Kim S, Blanchard E, et al. Biological aging and the human gut microbiota. J Gerontol A Biol Sci Med Sci. 2017; 72(11):1474-1482.

19. Jackson MA, Jeffery IB, Beaumont M, et al. Signatures of early frailty in the gut microbiota. Genome Med. 2016;8(1):8.

20. Jeffery IB, Lynch DB, O'Toole PW, et al. Composition and temporal stability of the gut microbiota in older persons. ISME J. 2016;10(1):170 82.

21. AK Vehmas, $\mathrm{CH}$ Kawas, WF Stewart, et al. Immune reactive cells in senile plaques and cognitive decline in Alzheimer's disease. Neurobiology of Aging. 2003;24(2):321-331.

22. D Giulian. Senile plaques stimulate microglia to release a neurotoxin found in Alzheimer brain. Neurochemistry International. 1995;27(1):119-137.

23. Beckman KB, Ames BN. The free radical theory of aging matures. Physiol Rev. 1998;78(2):547-581.

24. Dalile B, Oudenhove LV, Vervliet B, et al. The role of short-chain fatty acids in microbiota-gut-brain communication. Nature Reviews Gastroenterology \& Hepatology. 2019;16:461-478.

25. Bana B, Cabreiro F. The microbiome and aging. Annu Rev Genet. 2019;53:239-261. 